\title{
Gerenciamento de caso em ambulatório de psiquiatria, competências e prática da enfermeira
}

Recebido em: 14/03/2012

Aceito em: 30/01/2013

\author{
Ana Stella Silveira ${ }^{1}$ \\ Ana Carolina Siqueira ${ }^{2}$ \\ Fabiana de Mello Oliveira ${ }^{3}$ \\ Elizabeth Akemi Nishio ${ }^{4}$ \\ Maria do Perpétuo S.S. Nóbrega ${ }^{5}$
}

O presente artigo objetiva relatar a experiência da implantação do Gerenciamento de Caso em Ambulatório de Psiquiatria, descrevendo competências e práticas das enfermeiras. O modelo passou por diversas alterações até consolidar-se no atual. Cada enfermeira atua visando garantir continuidade e qualidade do tratamento através de estratégias de acolhimento, psicoeducação, elaboração e implementação do processo de enfermagem e do plano terapêutico multiprofissional. A taxa de abandono de tratamento correspondeu a 11\%, baixa quando comparada a estudos semelhantes. A experiência permitiu um cuidado coordenado e efetivo, além do aumento da autonomia das enfermeiras, aliado à responsabilização pelo cuidado longitudinal dispensado ao paciente. Descritores: Administração de Caso, Enfermagem Psiquiátrica, Papel do Profissional da Enfermagem.

\section{Case management in psychiatric ambulatory care, competences, and nursing practice}

This article aims to describe the experience of the implementation of Case Management in Psychiatric Ambulatory Care, the competences, and nursing practices. The model underwent many changes before assuming its current form. Each nurse works aiming at assuring the continuance and quality of the care through strategies of welcoming, psychoeducation, preparation, and implementation of the nursing process and the multi-professional therapeutic plan. The care abandonment rate corresponded to $11 \%$, which is low when compared to similar students. The experience allowed for a coordinated and effective care, besides increased autonomy of the nurses, related to the responsibility for longitudinal care to the patient.

Descriptors: Case Management, Psychiatric Nursing, Role of the Nursing Professional.

\section{Gerencia de caso en ambulatorio de psiquiatría, competencias y práctica de la enfermería}

El presente artículo tiene como objetivo relatar la experiencia de la implantación de la Gerencia de Caso en Ambulatorio de Investigación, describiendo competencias y prácticas de las enfermeras. El modelo pasó por diversas alteraciones hasta consolidarse en el actual. Cada enfermera actúa buscando garantizar la continuidad y la calidad del tratamiento a través de estrategias de acogida, psicoeducación, elaboración e implementación del proceso de enfermería y del plan terapéutico multiprofesional. La tasa de abandono de tratamiento correspondió al 11\%, baja cuando comparada a estudios semejantes. La experiencia permitió un cuidado coordinado y efectivo más allá del aumento de la autonomía de las enfermeras, aliado a la responsabilización por el cuidado longitudinal dispensado al paciente.

Descriptores: Administración de Caso, Enfermería Psiquiátrica, Papel del Profesional de Enfermería.

\section{INTRODUÇÃO}

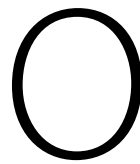

Gerenciamento de Caso (GC) é um "método de cuidado com um objetivo comum para toda a equipe de saúde na busca de resultados de qualidade para o paciente, família e membros envolvidos na assistência, com boa relação de custo-benefício, flexível a qualquer ambiente de cuidado, priorizando as populações que demandam assistência específica contínua e qualificada por um longo período de tempo"(1). O GC em Saúde Mental surgiu nos Estados Unidos na década de 1970 e é contemporâneo ao movimento de desospitalização, quando o fechamento de hospitais psiquiátricos resultou em elevadas taxas de reinternação, emergindo daí a necessidade da criação de estratégias mais efetivas para o tratamento comunitário como o GC. Este tem se mostrado um método efetivo para garantir a continuidade do tratamento para pacientes com transtornos mentais graves ${ }^{(2)}$. O GC em Psiquiatria contrapõe-se ao modelo tradicional hospitalocêntrico, pois busca tratar o paciente na comunidade e mantê-lo mais próximo do serviço de saúde ${ }^{(3)}$.

1 Enfermeira. Especialista em Enfermagem Psiquiátrica e Saúde Mental. Coordenadora de Enfermagem do Ambulatório Médico de Especialidades Jandira Masur (AMEJM)-Associação Paulista para o Desenvolvimento da Medicina. E-mail: anastella@gmail.com

2 Enfemeira. Especialista em Transtornos Alimentares. Supervisora de Enfermagem do AMEJM-Associação Paulista para o Desenvolvimento da Medicina.

3 Enfermeira. Especialista em Enfermagem Psiquiátrica e Saúde Mental. Supervisora de Enfermagem do AMEJM-Associação Paulista para o Desenvolvimento da Medicina.

4 Enfermeira. Mestre em Ciências pelo DIS-Unifesp. Coordenação e Direção de Enfermagem do AMEJM-Associação Paulista para o Desenvolvimento da Medicina.

5 Enfermeira. PhD em Dependência Química. Supervisora Educação Continuada em Enfermagem Psiquiátrica da Associação Paulista para o Desenvolvimento da Medicina (SPDM Afiliados). Docente do Curso de Graduação e Pós-Graduação em Enfermagem da FMABC. Representante do Conselho Federal de Enfermagem no Conselho Nacional de Drogas-CONAD-Brasil. 
As enfermeiras são profissionais constantemente escolhidas para atuar como Gerentes de Caso (GsC), uma vez que apresentam habilidades essenciais para o cumprimento desse papel, tais como: a capacidade de realizar múltiplas ações, a visão complexa do paciente, atenção aos detalhes, a capacidade para tomada de decisão, coordenação, liderança e comunicação(4). O GC difere da prática da enfermagem psiquiátrica convencional, em relação ao direcionamento de um plano de cuidado multidisciplinar, ao exigir o gerenciamento do cuidado de forma mais abrangente ${ }^{(5)}$. Quando aplicado consistentemente, esse método representa a implantação holística do processo de enfermagem, focado nas necessidades individuais dos pacientes ${ }^{(6)}$.

O Ambulatório Médico de Especialidades de Psiquiatria Dra. Jandira Masur (AMEPQ) é um serviço administrado pela Organização Social de Saúde (OSS), Associação Paulista para o Desenvolvimento da Medicina (SPDM), responsável pelo atendimento de pacientes residentes na Zona Norte do município de São Paulo. Consiste em um nível secundário de atenção e tem como objetivos otimizar o atendimento a pacientes com transtornos mentais de prevalência relevante na área de abrangência e complementar e fortalecer o atual sistema de atendimento em saúde mental ${ }^{(7)}$.

O objetivo deste artigo é relatar a experiência de implantação da tecnologia assistencial de GC em um serviço ambulatorial, e descrever as competências e a prática da enfermeira na aplicação dessa tecnologia.

\section{RELATO DE EXPERIÊNCIA}

$O$ atendimento no AMEPQ compreende as seguintes linhas de cuidado: Psicogeriatria, Psiquiatria infantil e da adolescência, Transtornos relacionados ao uso de álcool e drogas, Transtornos psicóticos e Transtornos afetivos e ansiosos. A assistência é prestada por equipe multiprofissional e inclui consultas individuais e grupos terapêuticos ou de família(7).

A duração do tratamento varia de acordo com o plano terapêutico do paciente, uma vez que o foco do serviço é a elucidação diagnóstica, estabilização do quadro e reorganização psíquica e funcional. Atingidos tais objetivos, realiza-se o encaminhamento do paciente à unidade de origem, como o Centro de Atenção Psicossocial, Unidade Básica de Saúde, ou seja, serviços de maior ou menor complexidade ${ }^{(7)}$.

Cada linha de cuidado do AMEPQ conta com ao menos duas enfermeiras que atuam como GsC. Assim, todos os pacientes admitidos no serviço são acompanhados por uma GsC com frequência e intensidade consonantes com o seu quadro clínico, psíquico e social.

A literatura aponta que não há uma padronização rígida do papel do GsC e que instituições que implementam esse modelo devem designá-lo conforme sua política, necessidades e objetivo. Destaca ainda que o GC deve incluir avaliação, coordenação e monitoramento do cuidado, como também de recursos humanos e materiais a fim de garantir a eficácia e individualização do atendimento(4).

Assim, a Diretoria de Enfermagem da SPDM Afiliados adotou o GC no AMEPQ e definiu as seguintes funções para as GsC:

1. Acompanhar o tratamento do paciente, desde a admissão até a alta: ter conhecimento e acolher todos os pacientes que são admitidos e planejar a alta junto à equipe multiprofissional e ao serviço social, a fim de que o paciente não fique desassistido até iniciar o tratamento na unidade para qual foi encaminhado;

2. Desenvolver o Processo de Enfermagem através das Consultas de Enfermagem de acordo com a Resolução $n^{\circ}$ 272/2002 do $\operatorname{COFEN}^{(8)}$;

3. Elaborar e implementar o plano terapêutico do paciente em conjunto com a equipe multiprofissional, incluindolevantamento de problemas, estabelecimento de metas e proposta de intervenções, atividades e encaminhamentos externos;

4. Participar e organizar a discussão clínica em sua linha de cuidado, reconhecendo e levantando previamente os casos de maior complexidade a serem discutidos;

5. Discutir o GC com a Supervisão e Educação Permanente em Enfermagem, através de apresentação de caso e/ou discussão de artigo científico a fim de se instrumentalizar

\begin{tabular}{|c|c|c|c|c|c|c|c|c|}
\hline Paciente & Nascimento & Admissão & Diagnóstico & GsC & Psiquiatra & Psicólogo/TO & $\begin{array}{l}\text { Observações } \\
\text { Importantes }\end{array}$ & $\begin{array}{c}\text { Encaminhado } \\
\text { para }\end{array}$ \\
\hline Joana & $8 / 5 / 79$ & $4 / 8 / 10$ & $\mathrm{~F} 20$ & Enf. Maria & David & Não passa & $\begin{array}{c}\text { Internada desde } \\
7 / 8 / 11\end{array}$ & Hospital X \\
\hline Amanda & $5 / 7 / 65$ & $10 / 5 / 10$ & F19 2 & Enf. Roberta & Matheus & Alice & Alta em 6/5/11 & Abandono \\
\hline Richard & $8 / 10 / 69$ & $6 / 12 / 10$ & F10 2 & Enf. Roberta & Matheus & Não passa & Alta em 6/5/11 & USBY \\
\hline Teresa & $11 / 10 / 67$ & $12 / 10 / 10$ & F60 & $\begin{array}{c}\text { Enf Maria } \\
.\end{array}$ & David & Alice & $\begin{array}{c}\text { Queixa de } \\
\text { efeitos colaterais }\end{array}$ & \\
\hline Victor & $7 / 7 / 88$ & $4 / 8 / 11$ & F29 & Enf. Maria & David & Não passa & $\begin{array}{l}\text { Presença de } \\
\text { familiar }\end{array}$ & \\
\hline
\end{tabular}

Quadro 1 - Modelo de Planilha de Gerenciamento de Caso 


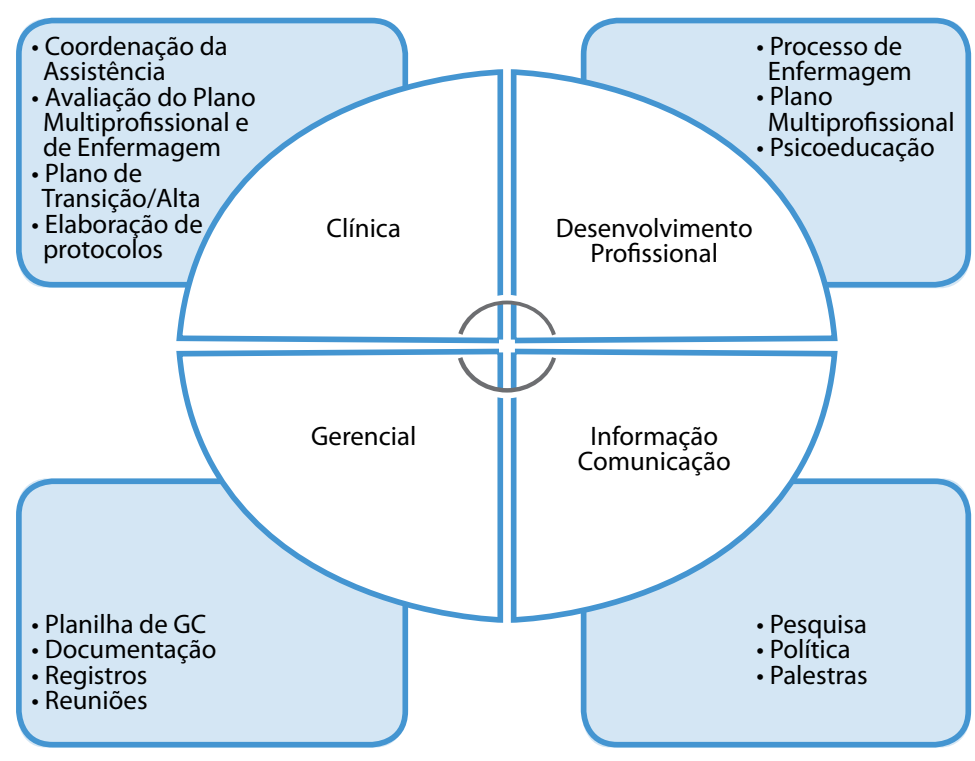

Figura 1 - Dimensões do Gerenciamento de Caso exercidas pela enfermeira no Ambulatório Médico de Especialidades Dra Jandira Masur.

para atender as demandas dos pacientes e do serviço, adquirindo mais segurança e autonomia em suas ações.

6. Organizar a Planilha de GC (Quadro1), condensando as principais informações observadas a respeito dos pacientes ou informadas pelos demais profissionais, por meio de contato direto, reuniões ou de um sistema interno de troca de informações;

7. Socializar com a equipe qualquer intercorrência ou alteração no quadro do paciente durante as reuniões e por meio da planilha de GC;

8. Reavaliar periodicamente o plano terapêutico do paciente; 9. Coordenar e delegar os cuidados com o paciente, fazendo cumprir os objetivos do plano terapêutico e supervisionando a execução da prescrição de enfermagem pelos técnicos.

10. Desenvolver atividades de psicoeducação para o paciente e

11. Garantir a qualidade e a continuidade do tratamento por meio de Busca Ativa (BA), estratégia de contato direto de profissional capacitado com o paciente faltoso, via telefone ou carta, para breve avaliação das condições clínicas e convite de retorno ao tratamento.

O exercício do GC no AMEPQ pode ser dividido em quatro dimensões, adaptadas de Cohen $2005^{(8)}$. A dimensão do GC mais tradicional na rotina da enfermeira é a clínica, que inclui o processo de enfermagem, o plano multiprofissional e atividades de psicoeducação. A dimensão inclui a promoção de atividades de ensino e coordenação do seguimento dos planos de enfermagem, multiprofissional e de alta, além da contribuição para a elaboração e implementação de protocolos. A dimensão informação/comunicação inclui atividades de registro e organização de dados em planilha, documentos e prontuários, além de trocas nas reuniões. A dimensão do desenvolvimento profissional implica na participação em pesquisas, elaboração de políticas e palestras intra e extrainstitucionais.
Após um ano da implantação do GC no AMEPQ um levantamento realizado demonstrou que cada enfermeira gerencia em média 228 pacientes, e que a taxa de abandono de tratamento foi de $11 \%$. Para obter tal dado, foram considerados abandonos de tratamento os casos que receberam alta por abandono, após faltarem à consulta médica e aos demais profissionais, e que não compareceram ao serviço para remarcar a consulta, mesmo após a realização de Busca Ativa via telefone ou carta registrada pelas GsC. A Tabela 1 representa os demais resultados assistenciais do GC no período de um ano (agosto de 2010 a julho de 2011).

\section{DISCUSSÃO}

O Gerenciamento de Casos foi implementado no AMEPQ na sua inauguração (agosto de 2010). No período de 12 meses, o modelo inicial passou por diversas alterações até consolidar-se no modelo atual, no qual o atendimento engloba cinco Linhas de Cuidado: Psicogeriatria, Psiquiatria infantil e da adolescência, Transtornos relacionados ao uso de álcool e drogas, Transtornos psicóticos e Transtornos afetivos e ansiosos. A GsC é responsável pela garantia da continuidade e qualidade do tratamento por meio de estratégias como acolhimento, psicoeducação, elaboração e implementação do processo de enfermagem e do plano terapêutico multiprofissional.

Algumas colocações são importantes acerca do modelo de GC no AMEPQ. Primeiramente, não há na literatura nacional recomendações a respeito do número ideal de pacientes a ser gerenciado por cada GsC. Autores recomendam que cada GsC assuma no máximo 50 casos, sendo dez casos o número ideal por $\mathrm{GsC}^{(4)}$. Mas, como o GC é flexível de acordo o serviço em que está inserido, o método no $A M E P Q$ permite que o número de casos por GsC seja maior que o recomendado pela literatura.

A experiência das autoras do presente artigo demonstrou que um número máximo de 100 casos por GsC seria o ideal para o GC no $A M E P Q$, que é um serviço destinado a atender pacientes moderados a graves durante o quadro agudo. Atualmente, a média de 228 casos/GsC reflete o aumento da demanda pelo serviço e a dificuldade em encaminhar pacientes estáveis para a Unidade de referência. Diante disso, mudanças estão sendo planejadas com o propósito de melhorar o fluxo de seguimento e a relação com a Rede de Saúde Mental.

As taxas de abandono de tratamento representaram $11 \%$ de todos os pacientes admitidos no AMEPQ durante um ano. Melhorar a adesão ao tratamento é um dos principais alvos do GC, mas a taxa de abandono do tratamento encontrada no AMEPQ pode ser considerada baixa quando comparada a outros estudos.

Pesquisa realizada em um Centro de Referência em Saúde Mental em Belo Horizonte encontrou taxa de abandono de $39,2 \%$ no período de um ano. Considerou-se abandono os casos de pacientes com indicação de retorno que não compareceram à unidade em até quatro meses ${ }^{(9)}$.

Estudo realizado nos Estados Unidos e em Ontário mostrou taxas de abandono de $19,2 \%$ e $16,9 \%$ respectivamente, sendo que foram significantemente menores para pacientes recebendo tratamento de dupla modalidade, ou seja, com um profissional médico e um não médico, do que para os pacientes tratados somente por um desses 
profissionais. Encontrou ainda que os pacientes que não consideravam o tratamento em Saúde Mental efetivo tiveram maiores taxas de abandono e sugeriu a psicoeducação como estratégia para adesão(10).

A baixa taxa de abandono identificada no AMEPQ pode estar relacionada ao fato de que o tratamento geralmente compreende mais uma modalidade de atendimento, além do atendimento médico e Gerenciamento de Caso, dispensados a todos os pacientes. Ademais, as enfermeiras $\mathrm{GsC}$ são responsáveis por realizar Busca Ativa para os faltosos e pela psicoeducação individual ou em grupo para pacientes e família, promovendo o entendimento do processo de adoecimento e tratamento.

O presente estudo apresenta como limitação o método utilizado para medir a taxa de abandono. Este foi realizado

Tabela 1 - Resultados referentes às Linhas de Cuidado

\begin{tabular}{|l|c|c|c|}
\hline Linha de cuidado & $\begin{array}{c}\text { Número de } \\
\text { Gerentes } \\
\text { de Caso }\end{array}$ & $\begin{array}{c}\text { Pacientes } \\
\text { gerenciados } \\
\text { por Gerente } \\
\text { de Caso }\end{array}$ & $\begin{array}{c}\text { Taxa de } \\
\text { Abandono }\end{array}$ \\
\hline $\begin{array}{l}\text { Transtorno de álcool e } \\
\text { outras drogas }\end{array}$ & 2 & 110 & $30 \%$ \\
\hline $\begin{array}{l}\text { Transtornos psicóticos } \\
\text { Transtornos afetivos e } \\
\text { ansiosos }\end{array}$ & 2 & 226 & $2 \%$ \\
\hline $\begin{array}{l}\text { Psicogeriatria } \\
\text { Psiquiatria infantil }\end{array}$ e da adolescência & 2 & 361 & $10 \%$ \\
\hline Média & 2 & 144 & $13 \%$ \\
\hline
\end{tabular}

Resultados referentes às Linhas de Cuidado: pacientes gerenciados por Gerente de Caso e taxa de abandono correspondentes à média do período de agosto de 2010 a julho de 2011. retrospectivamente e considerou a anotação em planilha de GC, seguindo o critério de alta por abandono estabelecido no AMEPQ, o que pode ter gerado uma subnotificação dos casos.

As taxas de abandono por Linha de Cuidado variaram entre 2 (Psicogeriatria e Psicose) a 30\% (Álcool e Drogas). A clientela da Linha de Cuidado de Álcool e Drogas apresenta um perfil de ambivalência em relação ao tratamento, comprometendo a adesão, o que exige a intensificação de ações voltadas para a motivação. Os pacientes das Linhas Psicogeriatria e Psicose aderem mais ao tratamento, possivelmente pelo comprometimento clínico, e por encontrar no serviço um espaço de socialização e acolhimento para os mesmos e seus cuidadores.

Na Linha da Infância e Adolescência, a taxa de 13\% reflete o balanço entre a dificuldade da aceitação da doença e o trabalho intensivo de aproximação e responsabilização dos pais com o tratamento. A clientela da Linha de Transtornos Afetivos e Ansiosos apresenta característica de resposta rápida ao tratamento e melhor funcionamento social, o que pode conduzir ao abandono precoce.

\section{CONCLUSÃO}

A experiência do GC no AMEPQ durante esses 12 meses permitiu o cuidado mais coordenado e efetivo, diminuindo entraves como a perda da continuidade e a comunicação inadequada. Nesse período, as enfermeiras tiveram que lidar com um novo modelo assistencial, que exige uma prática mais autônoma, com responsabilização pelo cuidado longitudinal dispensado ao paciente.

O AMEPQ foi o primeiro serviço da SPDM a implementar o modelo de GC, e hoje expande-se para outras unidades como os Centros de Atenção Psicossocial(CAPS) e Unidades de internação em Psiquiatria em Hospital Geral. A Gestão de Enfermagem da SPDM compreende que a adoção de modelos de trabalho cientificamente comprovados é necessária para a consolidação da prática de enfermagem para garantir a assistência de qualidade.

\section{Referências}

1. Casarin S, Villa TCS, Cardozo-Gonzales Rl, Caliri MHL, Freitas MC. Gerenciamento de

caso: análise de conceito. Invest Educ Enferm. 2003;21(1):26-36.

2. Marshall M, Gray A, Lockwood A, Green R. Withdrawn: case management for people with severe mental disorders. Cochrane Database System Rev [internet]. 2011;(4):CD000050. Disponível em: http://www.update-software.com/BCP/ WileyPDF/EN/CD000050.pdf.

3. Santos AM, Luis MAV. Gerenciamento de casos como estratégia de trabalho para a enfermagem psiquiátrica comunitária. Rev Esc Enferm USP. 2005;39(2):235.

4. Cohen EL, Cesta TG, editoras. Nursing case management: from essentials to

advanced practice applications. $4^{\mathrm{a}}$ ed. Philadelphia: Elsevier; 2005.

5. Soares MH. Conhecimentos básicos no gerenciamento de casos de saúde mental

[Internet]. 2009;5(2). Disponível em: http://www.revistasusp.sibi.usp.br/scielo.

php?script=sci_arttext\&pid=S1806-69762009000200010\&lng=pt.
6. McCullough L. The case manager: an essential link in quality care. Creat Nurs. 2009;15(3):124-26.

7. De Araújo Filho GM, Amino D, Yamaguchi LM, Silveira ASA, Tamai S. AME Psiquiatria Vila Maria e sua contribuição para a assistência à saúde mental: dados referentes ao primeiro ano de funcionamento. Rev Debates Psiquiatr. 2012;2:44-9. 8. Brasil. Resolução do Conselho Federal de Enfermagem 272/2002. Regulamenta a Sistematização da Assistência de Enfermagem (SAE) nas instituições de saúde brasileiras. Rio de Janeiro: 2002.

9. Melo APS, Guimarães MDC. Factors associated with psychiatric treatment dropout in a mental health reference center, Belo Horizonte. Rev Bras Psiquiatr. 2005;27(2):113-18. 10. Edlund MJ, Wang PS, Berglund PA, Katz SJ, Lin E, Kessler RC. Dropping out of mental health treatment: patterns and predictors among epidemiological survey respondents in the United States and Ontario. Am J Psychiatry. 2002;159:845-85. 